\title{
Cone-Beam Tomography with Linearly Distorted Source Trajectories
}

\author{
Frank Dennerlein, Student Member, IEEE, Frédéric Noo, Member, IEEE, Stefan Hoppe, Student Member, IEEE, \\ Joachim Hornegger, and Günter Lauritsch
}

\begin{abstract}
A major drawback of Katsevich's exact general cone-beam inversion scheme is the difficulty in finding practical algorithms adapted to every novel type of source trajectory. We succeeded to overcome this problem, and can formulate reconstruction algorithms, for trajectories that are related through a linear distortion to an already studied scenario. The introduced theory yields two reconstruction strategies that are in principle independent from the underlying reconstruction algorithm and are either based on data pre- and post-processing or on adjustment of filtering directions. Numerical results based on simulated cone-beam data are presented.
\end{abstract}

Index Terms - Computed tomography, exact cone-beam reconstruction, linearly distorted source trajectories

\section{INTRODUCTION}

In recent years, computed tomography (CT), in particular three-dimensional (3D) cone-beam (CB) reconstruction theory has undergone significant advances. As a major breakthrough, Katsevich introduced a general scheme to find exact $\mathrm{CB}$ inversion formulas for most complete source trajectories [1]. One essential component of this inversion scheme is an auxiliary function that has to be defined and adjusted to the actual acquisition scenario in order to obtain a practical and efficient reconstruction algorithm. The attribute "practical" refers here to the algorithm allowing a good amount of data truncation, and thus requiring little $\mathrm{X}$-ray exposure outside the imaged slab of the patient body. Unfortunately, finding a good auxiliary function is in general a very intellectual process requiring significant effort. To do so, one typically needs to carefully study how planes through each object point within the region-of-interest (ROI) intersect various segments of the source trajectory. The auxiliary function defines how to weight data on these planes within the reconstruction process. Only some auxiliary functions allow data truncation, and these functions only exist for specific segments of the source trajectory that generally depend on the object point. Using his theory in [1], Katsevich investigated several trajectories that are relevant for real world CT devices, such as the medical $\mathrm{CT}$ scanner or the $\mathrm{C}$-arm device. He found auxiliary functions

F. Dennerlein and F. Noo are with the Department of Radiology, University of Utah, Salt Lake City, Utah, USA

E-mail: fdenner@ucair.med.utah.edu

S. Hoppe and J. Hornegger are with the Institute of Pattern Recognition, University of Erlangen-Nuremberg, Erlangen, Germany

G. Lauritsch is with Siemens AG, Medical Solutions, Forchheim, Germany.

This work was partially supported by a grant of Siemens AG, Medical Solutions and by the U.S. National Institutes of Health (NIH) under grant R01 EB000627. Its contents are solely the responsibilitiy of the authors and do not necessarily represent the official views of the NIH. that yield practical, accurate algorithms for the ideal helical trajectory [2], the ideal circle-plus-(orthogonal)-arc [3], and the ideal circle-plus-(orthogonal)-line trajectory [4]. However, the algorithms are restricted to the source trajectories they were derived for. Therefore, they do not readily apply to, for example, a source trajectory made of a circle and an oblique arc, which is a simple deviation from the ideal circle-plusarc trajectory. In [5], Noo et al. presented a data rebinning strategy to easily obtain a reconstruction algorithm for a helix-with-gantry-tilt trajectory from algorithms derived for a conventional (non-tilted) helix. In [6], the authors also applied this rebinning method to allow reconstruction from another specific trajectory. In this article, we apply that concept to obtain theoretically-exact reconstruction approaches for a large spectrum of novel and unconventional acquisition geometries. Instead of deriving algorithms from the general CB inversion scheme, we propose a theory which allows the development of practical and accurate $\mathrm{CB}$ reconstruction algorithms for source trajectories that differ from an already studied, so called ideal scenario by a linear transformation. From that theory, we derive two reconstruction approaches that utilize an existing implementation of an algorithm for the ideal scenario. These approaches consist of (i) a direct data rebinning strategy in a pre- and post processing step or (ii) a modification of filtering directions within the filtered-backprojection framework of the available implementation.

\section{Modeling of Geometry Distortions}

Consider a source trajectory for which a $\mathrm{CB}$ reconstruction algorithm has been implemented. Let $\lambda$ be the parameter describing this trajectory and $\underline{a}_{0}(\lambda) \in \mathbb{R}^{3}$ be the source position at a given $\lambda$. The trajectory $\underline{a}_{0}(\lambda)$ may be a smooth curve like the ideal helical trajectory, or may be the union of several smooth curves, like the ideal circle-plus-arc trajectory. CB data acquisition along a path $\underline{a}_{0}(\lambda)$ will be referred to as an ideal scenario or just ideal throughout this article.

We can now introduce a linearly distorted source trajectory $\underline{a}(\lambda)$ that is related to an ideal path by

$$
\underline{a}(\lambda)=A \underline{a}_{0}(\lambda), \quad A \in \mathbb{R}^{3 \times 3}, \quad \operatorname{det} A \neq 0,
$$

with the non-singular matrix $A$ describing a bijective linear mapping in $\mathbb{R}^{3}$. The distortion defined by (1) operates globally on the ideal trajectory so that, if $\underline{a}(\lambda)$ is composed of several smooth segments, all of them are distorted by the same operation $A$. Although any linear transformation may be considered, shearing operations and non-isotropic scaling 
are of special interest. Then, the curves $\underline{a}_{0}(\lambda)$ and $\underline{a}(\lambda)$ differ in shape so that a distinct acquisition scenario is obtained and with that we get a different $\mathrm{CB}$ reconstruction problem. To get a rough idea about potential effects of (1) onto an ideal trajectory, we use the fact that certain geometric properties are invariant under a linear transformation. For instance, if a segment of the ideal source trajectory is entirely located in a plane, then the corresponding segment in $\underline{a}(\lambda)$ will be planar, too. Also, a line in $\underline{a}_{0}(\lambda)$ is mapped onto a line in $\underline{a}(\lambda)$, and if $\underline{a}_{0}(\lambda)$ consists of several connected segments, these segments will remain connected to each other in $\underline{a}(\lambda)$.

\section{RECONSTRUCTION THEORY}

CB data acquired along a source trajectory $\underline{a}(\lambda)$ consists of line integrals that may be written as

$$
g(\lambda, \underline{\theta})=\int_{0}^{\infty} d t f(\underline{a}(\lambda)+t \underline{\theta}),
$$

where $f(\underline{x})$ with $\underline{x} \in \mathbb{R}^{3}$ describes the spatial distribution of the linear X-ray attenuation coefficient, while $\underline{\theta}$ is a unit vector giving the line direction. Now, if $\underline{a}(\lambda)$ is related to an ideal trajectory, we can substitute (1) into (2) and introduce $\underline{\alpha}=\left(A^{-1} \underline{\theta}\right) /\left(\left\|A^{-1} \underline{\theta}\right\|\right)$ to get

$$
\begin{aligned}
g(\lambda, \underline{\theta}) & =\int_{0}^{\infty} d t f\left(A \underline{a}_{0}(\lambda)+t \underline{\theta}\right) \\
& =\int_{0}^{\infty} d t f\left(A\left(\underline{a}_{0}(\lambda)+t A^{-1} \underline{\theta}\right)\right) \\
& =\int_{0}^{\infty} d t f_{v}\left(\underline{a}_{0}(\lambda)+t A^{-1} \underline{\theta}\right) \\
& =\frac{1}{\left\|A^{-1} \underline{\theta}\right\|} g_{v}(\lambda, \underline{\alpha}) .
\end{aligned}
$$

Here, $f_{v}(\underline{x})=f(A \underline{x})$ denotes a virtual object, which is a distorted version of the object under investigation. Equation (3) establishes a link between the acquired line integrals $g(\lambda, \underline{\theta})$ and CB data $g_{v}(\lambda, \underline{\alpha})$ of the virtual object, which corresponds to an ideal source trajectory. The inverse relations are given as

$$
f(\underline{x})=f_{v}\left(A^{-1} \underline{x}\right)
$$

and

$$
g_{v}(\lambda, \underline{\theta})=\frac{1}{\|A \underline{\theta}\|} g\left(\lambda, \frac{A \underline{\theta}}{\|A \underline{\theta}\|}\right) .
$$

\section{Reconstruction USING DiRECT DATA REBINNING}

From the presented theory, a three step reconstruction method can be readily formulated:

(i) Rebinning of CB data at fixed $\lambda$

Using (5) we obtain CB data of the virtual object $f_{v}(\underline{x})$ for the corresponding ideal source trajectory $\underline{a}_{0}(\lambda)$.

(ii) Reconstruction of the virtual object

Using any existing reconstruction algorithm for the ideal trajectory $\underline{a}_{0}(\lambda)$, we can reconstruct $f_{v}(\underline{x})$ from the rebinned CB data $g_{v}(\lambda, \underline{\alpha})$ created in step (i).

(iii) $3 \mathrm{D}$ rebinning in the image volume
The desired values of $f(\underline{x})$ are obtained from the reconstructed $f_{v}(\underline{x})$ by a $3 \mathrm{D}$ rebinning step according to (4).

Note that the 3D interpolation required in step (iii) comes with a loss in high frequency, so that the sought object function $f(\underline{x})$ is of lower spatial resolution compared to $f_{v}(\underline{x})$. However, if the implemented reconstruction algorithm allows modifications in the backprojection functionality, one can beneficially adjust the sampling grid on which the virtual object is reconstructed. Instead of using a Cartesian grid, the values of $f_{v}$ are obtained immediately at the sampling points $A^{-1} \underline{x}$. Then, a subsequent interpolation to obtain $f(\underline{x})=$ $f_{v}\left(A^{-1} \underline{x}\right)$ can be avoided and step (iii) becomes obsolete. This approach is referred to as the improved rebinning method.

\section{Reconstruction using Filtering Line ADJUSTMENT}

From the theory presented in Sec. III, a CB reconstruction algorithm can be derived that comes along without any additional data rebinning steps. This derivation may be applied to any filtered-backprojection reconstruction formula that is based on the concept of $\pi$-lines and filtering planes. Here, we consider Katsevich's theoretically-exact CB reconstruction algorithms for the ideal-circle-plus-arc and ideal-circle-plusline trajectory, respectively, both derived from the general CB inversion scheme [1].

From (4) we know that the reconstruction of $f(\underline{x})$ in the distorted acquisition set-up is identical to the computation of the density of the virtual object $f_{v}$ at point $A^{-1} \underline{x}$ in the corresponding ideal scenario. We observe further that the $\pi$-line determined for $A^{-1} \underline{x}$ in the ideal scenario (which intersects the trajectory at $\underline{a}_{0}\left(\lambda_{i}\right)$ and $\left.\underline{a}_{0}\left(\lambda_{o}\right)\right)$ is mapped onto a $\pi$-line for $\underline{x}$ in the linearly distorted scenario; this $\pi$-line intersects the distorted trajectory at $\underline{a}\left(\lambda_{i}\right)$ and $\underline{a}\left(\lambda_{o}\right)$, i.e. at unchanged curve parameters. Consequently, if the reconstruction of $f_{v}\left(A^{-1} \underline{x}\right)$ from CB data on $a_{0}(\lambda)$ is obtained through backprojection over the interval $\left[\lambda_{i}, \lambda_{o}\right]$ as in [3] or [4], so may the reconstruction of $f(\underline{x})$ from data on $\underline{a}(\lambda)$. The point that needs to be addressed is the data filtering.

With the existing algorithms [3] and [4] we can determine the filtering planes required for a reconstruction at $A^{-1} \underline{x}$ in the ideal scenario. Consider now an arbitrary filtering plane $P_{0}$. It intersects with the trajectory at $\underline{a}_{0}\left(\lambda_{p}\right)$ with $\lambda_{i} \leq \lambda_{p} \leq$ $\lambda_{o}$, contains by definition the point $A^{-1} \underline{x}$ and has normal vector $\underline{n}_{0}$. Geometrically, the linear distortion with $A$ yields a mapping of $P_{0}$ onto the plane $P$ on which filtering has to be performed in the distorted scenario. The plane $P$ contains the source point $\underline{a}\left(\lambda_{p}\right)=A \underline{a}_{0}\left(\lambda_{p}\right)$, the point $\underline{x}=A A^{-1} \underline{x}$ and is orthogonal to the vector $\underline{n}=A^{-T} \underline{n}_{0} /\left\|A^{-T} \underline{n}_{0}\right\|$. Therefore, it specifies the filtering direction for $\mathrm{CB}$ data acquired in the distorted scenario at curve parameter $\lambda_{p}$ required for a reconstruction at the point $\underline{x}$. The mathematics of this approach have already been derived but are left out for the purpose of conciseness.

When assuming a flat panel detector geometry, this one-onone mapping of filtering planes corresponds to a transformation of (ideal) filtering lines that are determined for the ideal 
scenario onto filtering lines required for $\mathrm{CB}$ projection images occurring in the distorted acquisition geometry. Modification of the filtering directions makes a major difference to the rebinning method of Sec. IV. There, the ideal filtering lines were used and an initial rebinning of $\mathrm{CB}$ data accounted for the distorted trajectory. In this second approach, however, acquired CB data is not modified but we adjust the filtering lines appropriately and allow thereby efficient and accurate reconstruction without the drawbacks of data rebinning. Using this filtering line adjustment strategy, we expect an improved spatial resolution in the reconstructions compared to the method of section IV.

\section{NUMERICAL RESULTS}

The two suggested reconstruction approaches for linearly distorted source trajectories were evaluated using computersimulated CB data of two analytically defined phantoms, the FORBILD head phantom [7] and a disc phantom, which is a variation of the well-known Defrise phantom. Numerical studies assume a flat detector geometry, where X-ray sensitive detector elements are aligned on a 2D Cartesian grid. We considered two distinct data acquisition set-ups, for which no direct reconstruction algorithm has been derived to our knowledge.

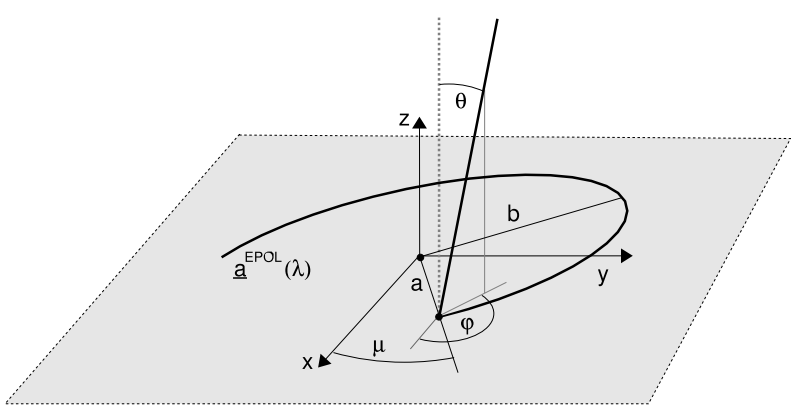

Fig. 1. The ellipse-plus-oblique-line trajectory $\underline{a}^{E P O L}(\lambda)$. The dimensions of the ellipse are defined by the half axes $a$ and $b$ and its rotation is given by the angle $\mu$. The linear scan segment is attached non-orthogonally to an endpoint of the ellipse and describes an angle of $\pi / 2-\theta$ with the plane of the elliptical scan.

First, data acquisition for an ellipse-plus-oblique-line trajectory $\underline{a}^{E P O L}(\lambda)$ was simulated. The elliptical segment covers

TABLE I

PROJECTION DATA SIMULATION PARAMETERS

\begin{tabular}{|c|c|c|}
\hline & $\begin{array}{l}\text { ellipse-plus } \\
\text { oblique-line }\end{array}$ & $\begin{array}{l}\text { circle-plus } \\
\text { oblique-arc }\end{array}$ \\
\hline $\begin{array}{l}\text { detector discretization } \\
\text { source-to-detector distance }\end{array}$ & $\begin{array}{c}0.4 \times 0.4 \mathrm{~mm}^{2} \\
80 \mathrm{~cm}\end{array}$ & $\begin{array}{c}0.4 \times 0.4 \mathrm{~mm}^{2} \\
80 \mathrm{~cm}\end{array}$ \\
\hline $\begin{array}{l}\text { properties of ellipse/circle } \\
\text { dimensions } \\
\text { orientation } \\
\text { discretization } \\
\text { \# of projections (head/disc) }\end{array}$ & $\begin{array}{c}a=88 \mathrm{~cm} \\
b=80 \mathrm{~cm} \\
\mu=0^{\circ} \\
\Delta \lambda=0.4^{\circ} \\
496 / 496\end{array}$ & $\begin{array}{c}R=80 \mathrm{~cm} \\
- \\
\Delta \lambda=0.4^{\circ} \\
497 / 498\end{array}$ \\
\hline $\begin{array}{l}\text { properties of line/arc } \\
\text { radius } \\
\text { orientation } \\
\text { discretization } \\
\text { \# of projections (head/disc) }\end{array}$ & $\begin{array}{c}\theta=10^{\circ}, \varphi=45^{\circ} \\
\Delta z=1 \mathrm{~mm} \\
167 / 333\end{array}$ & $\begin{array}{c}R=80 \mathrm{~cm} \\
\mu=-10^{\circ} \\
\Delta \lambda=0.4^{\circ} \\
31 / 60\end{array}$ \\
\hline
\end{tabular}

an angular interval of length $\lambda_{\text {ellipse }}$ and is located in the plane $z=0 \mathrm{~mm}$. The ellipse dimensions are defined by the half axis $a$ and $b$ and its orientation is described by the angle $\mu$, measured between the $x$-axis and the direction of the half axis with length $a$. The linear scan segment is attached to an endpoint of the ellipse such that it describes an angle of $\pi / 2-\theta$ with the plane of the elliptical scan. The azimuthal angle of the line is $\varphi$. See Fig. 1 for an illustration of the acquisition set-up. The shape of the considered trajectory suggests to relate it to the ideal circle-plus-line trajectory, which can be parametrized as

$$
\underline{a}_{0}^{I C P L}(\lambda)= \begin{cases}{[R, 0,(\lambda l) /(2 \pi)]^{T}} & \text { if } 0 \leq \lambda<2 \pi \\ {[R \cos \lambda, R \sin \lambda, 0]^{T}} & \text { if } 2 \pi \leq \lambda<2 \pi+\lambda_{c} .\end{cases}
$$

Here, $R$ denotes the radius and $\lambda_{c}$ the angular interval of the ideal circle scan and $l$ the length of the orthogonally attached line segment. We can determine a linear transform $A_{1}$ consisting of subsequent $3 \mathrm{D}$ shearing, non-isotropic scaling and rotation operations as

$$
A_{1}=\left[\begin{array}{ccc}
a^{\prime} \cos \mu & -b^{\prime} \sin \mu & \sin \theta \cos \varphi \\
a^{\prime} \sin \mu & b^{\prime} \cos \mu & \sin \theta \sin \varphi \\
0 & 0 & \cos \theta
\end{array}\right]
$$

such that $\underline{a}^{E P O L}(\lambda)=A_{1} \underline{a}_{0}^{I C P L}(\lambda)$. Here, $\lambda_{\text {ellipse }}=\lambda_{c}$ and $a^{\prime}$ and $b^{\prime}$ are selected so that $a=a^{\prime} R$ and $b=b^{\prime} R$.

In a second evaluation, we considered data acquisition along a circle-plus-oblique-arc trajectory $\underline{a}^{C P O A}(\lambda)$, which consists of two circular segments with common center and identical radius $R$. The circle scan is located in the plane $z=0 \mathrm{~mm}$ and covers an angular interval of length $\lambda_{c}$. The plane of the arc scan contains the $\mathrm{x}$-axis and is rotated by an angle $\theta$ from its vertical position. The length of the arc segment is $\lambda_{a}$. The two segments are connected to each other in their endpoint that has $z=y=0 \mathrm{~mm}$. For a geometric illustration of the acquisition set-up see Fig. 2. The described trajectory is a variation of the ideal circle-plus-arc path, which is given by

$$
\underline{a}_{0}^{I C P A}(\lambda)= \begin{cases}{[R \cos \lambda, 0, R \sin \lambda]^{T}} & \text { if } 0 \leq \lambda<\lambda_{a}, \\ {[R \cos \lambda, R \sin \lambda, 0]^{T}} & \text { if } 2 \pi \leq \lambda<2 \pi+\lambda_{c} .\end{cases}
$$

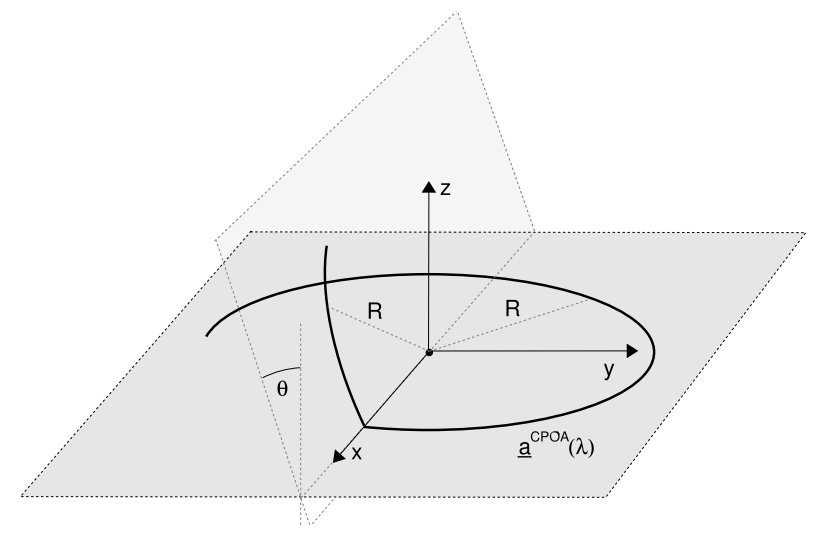

Fig. 2. The circle-plus-oblique-arc trajectory $\underline{a}^{C P O A}(\lambda)$. The two scan segments are both of circular shape with radius $\bar{R}$ and are located in planes that contain the $x$-axis. 
The linear transform

$$
A_{2}=\left[\begin{array}{ccc}
1 & 0 & 0 \\
0 & 1 & \sin \theta \\
0 & 0 & \cos \theta
\end{array}\right]
$$

maps the ideal trajectory $\underline{a}_{0}^{I C P A}(\lambda)$ onto $\underline{a}^{C P O A}(\lambda)$ according to $(1)$.
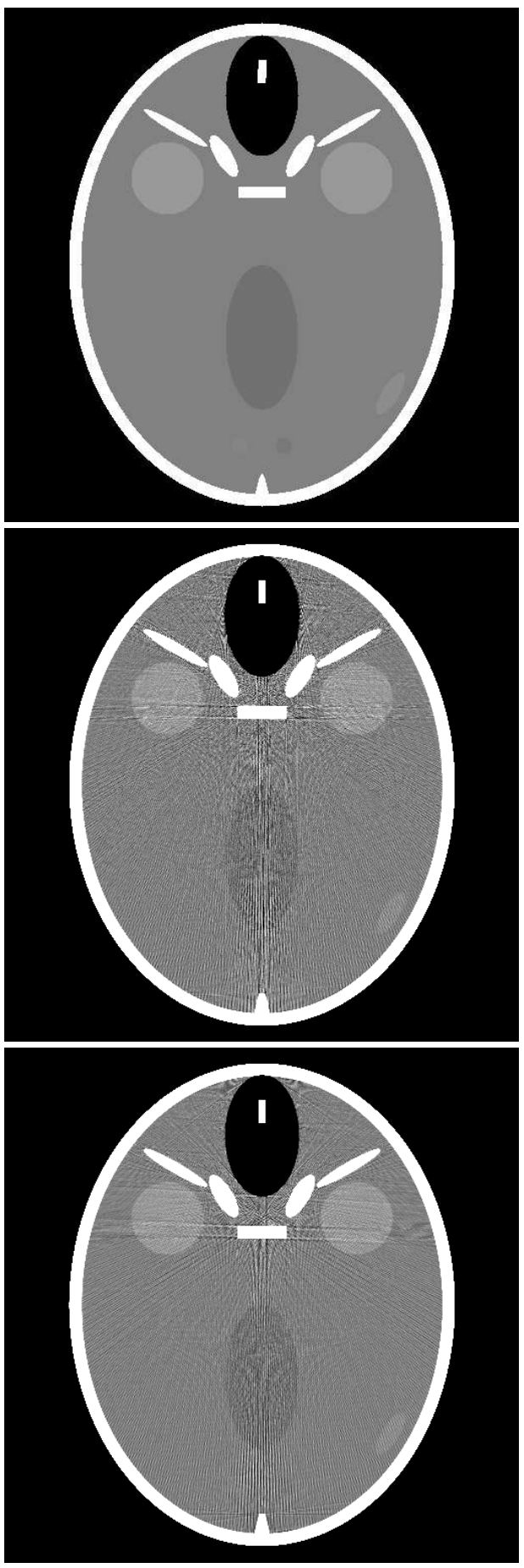

Fig. 3. Transaxial slice $z=40 \mathrm{~mm}$ of the FORBILD head phantom (centered around $z=40 \mathrm{~mm}$ ) in the grayscale window $[0,100] \mathrm{HU}$ : (top) values of $f(\underline{x})$ from analytical phantom definition, (center) reconstruction of $f(\underline{x})$ from $\mathrm{CB}$ data corresponding to $\underline{a}^{E P O L}(\lambda)$, (bottom) $f(\underline{x})$ obtained from $\mathrm{CB}$ data for $\underline{a}^{C P O A}(\lambda)$. For both reconstructions, the filtering line adjustment method was used.
The two investigated reconstruction scenarios involve trajectories which have just been identified as linearly distorted versions of previously studied, ideal trajectories with distortion parameters $A_{1}$ and $A_{2}$, respectively. Therefore, the object function $f(\underline{x})$ can be reconstructed using the algorithms described in Sec. IV and Sec. V.

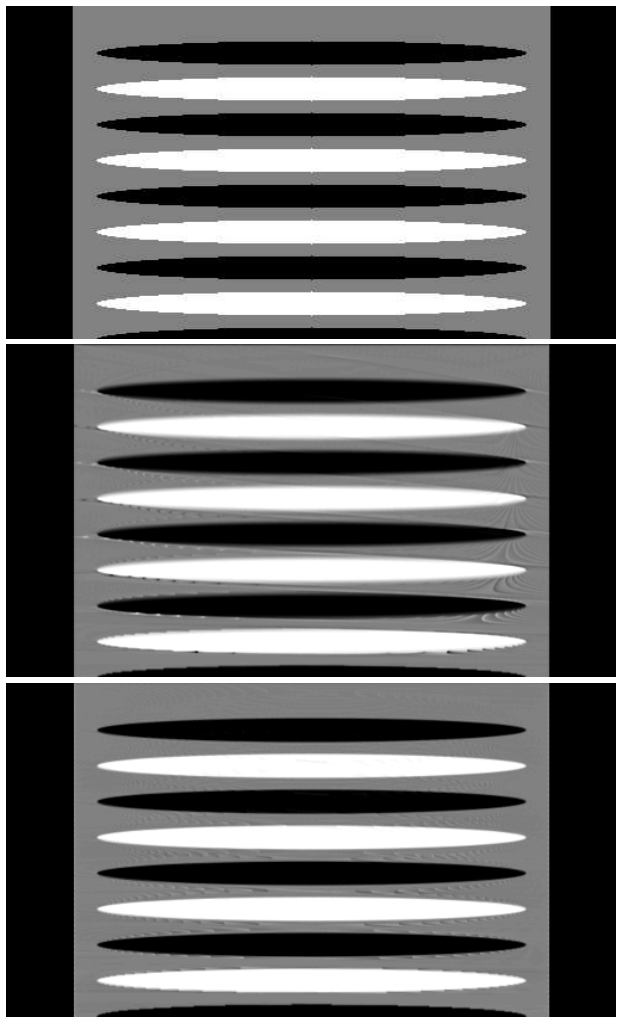

Fig. 4. Slice $x=0 \mathrm{~mm}$ for $z \in[0,140] \mathrm{mm}$ of the disc phantom using the grayscale window $[-500,500] \mathrm{HU}$ : (top) values of $f(\underline{x})$ from the phantom definition, (center) result obtained for the ellipse-plus-oblique-line trajectory and (bottom) for the circle-plus-oblique-arc trajectory. Both images were reconstructed using the improved rebinning method.

For both trajectories, the extent of the scan segments was selected large enough to guarantee a complete set of CB data for a theoretically-exact reconstruction of the region of interest. Also, detector position and dimensions were chosen to yield transaxially untruncated projection data. A comprehensive list of simulation parameters is presented in table I. Core CB reconstruction was carried out using our implementation of the accurate filtered-backprojection algorithms proposed by Katsevich for the ideal circle-plus-line [4] (for evaluation 1) and the ideal circle-plus-arc trajectory [3] (for evaluation 2).

The values of $f(\underline{x})$ were obtained on a Cartesian grid of cubic voxels of size $0.5 \times 0.5 \times 0.5 \mathrm{~mm}^{3}$. Fig. 3 presents image results for the FORBILD head phantom in a narrow grayscale window of width $100 \mathrm{HU}$. An off-center transaxial slice through the reconstructions of the phantom is visualized. The images were obtained from $\mathrm{CB}$ data corresponding to $\underline{a}^{E P O L}(\lambda)$ and $\underline{a}^{C P O A}(\lambda)$ using the filtering line adjustment reconstruction approach of section $\mathrm{V}$. The results are presented next to the corresponding slice of the original phantom.

Reconstructions of the disc object from CB data corresponding to the trajectories $\underline{a}^{E P O L}(\lambda)$ and $\underline{a}^{C P O A}(\lambda)$ were obtained 
using the improved rebinning method of IV. A central vertical slice through the image results is presented in Fig. 4 against the original phantom definition using a grayscale window of width $1000 \mathrm{HU}$.

\section{Conclusions}

We presented two strategies that allow $\mathrm{CB}$ reconstruction from a large class of (novel) source trajectories that are related to any well-studied acquisition scenario by a linear distortion. The approaches can be understood as extensions to already available algorithms, and in principle, every filteredbackprojection style algorithm can be applied for actual reconstruction. During simulation studies, we were able to achieve high quality image reconstructions from an ellipseplus-oblique-line trajectory by involving two theoreticallyexact reconstruction algorithms arising from Katsevich's general CB inversion scheme. To our knowledge, another accurate reconstruction approach [8] can deal with distorted source trajectories more naturally. However, this algorithm may have drawbacks in terms of data utilization and detector requirements compared to the presented strategies. This issue will be investigated more closely in the future.

The presented reconstruction approach is applicable to existing medical devices, since a linearly distorted trajectory may represent the scanning motion of an X-ray imaging device better than currently investigated ideal paths $\underline{a}_{0}(\lambda)$.

\section{REFERENCES}

[1] A. Katsevich, "A general scheme for constructing inversion algorithms for cone beam CT," Int. J. Math. Math. Sci., vol. 21, pp. 1305-1321, 2003.

[2] — "An inversion algorithm for spiral CT," in Proceedings Int. Conf. on Sampling Theory and Applications, 2001, pp. 261-5.

[3] — "Image reconstruction for the circle and arc trajectory," Phys. Med. Biol., vol. 50, pp. 2249-2265, 2005.

[4] - "Image reconstruction for the circle and line trajectory," Phys. Med. Biol., vol. 49, pp. 5059-5072, 2004.

[5] F. Noo, M. Defrise, and H. Kudo, "General reconstruction theory for multislice X-ray computed tomography with a gantry tilt." IEEE Trans. Med. Imaging, vol. 23, no. 9, pp. 1109-1116, 2004.

[6] S. L. T. Zhuang, B. E. Nett and G. H. Chen, "A shift-invariant filtered backprojection (FBP) cone-beam reconstruction algorithm for the source trajectory of two concentric circles using an equal weighting scheme," Phys. Med. Biol. 51, pp. 3189-3210, 2006.

[7] "Description of the FORBILD Head Phantom, online at http://www.imp.uni-erlangen.de/forbild/english/results/index.htm."

[8] J. D. Pack and F. Noo, "Cone-beam reconstruction using 1D filtering along the projection of M-lines," Inverse Problems, vol. 21, pp. 11051120,2005 